\title{
Recurrent Lobar Torsion
}

\author{
Hassan Alghamdi \\ Dalhousie University, Halifax, Canada. \\ Email: dr_hassan1117@yahoo.com
}

Received May 23 ${ }^{\text {rd }}$, 2013; revised June $23^{\text {rd }}$, 2013; accepted June $30^{\text {th }}, 2013$

Copyright (c) 2013 Hassan Alghamdi. This is an open access article distributed under the Creative Commons Attribution License, which permits unrestricted use, distribution, and reproduction in any medium, provided the original work is properly cited. In accordance of the Creative Commons Attribution License all Copyrights (C) 2013 are reserved for SCIRP and the owner of the intellectual property Hassan Alghamdi. All Copyright (C) 2013 are guarded by low and by SCIRP as a guardian.

\begin{abstract}
We report a first case of $180^{\circ}$ left upper lobe lung torsion followed by a $90^{\circ}$ lobar torsion in the same lobe after a left lower lobectomy.
\end{abstract}

Keywords: Complication; Lobar; Left Upper Lobe; Recurrent; Torsion

\section{Introduction}

Lung torsion is a rare but potentially lethal complication with an incident rate of approximately $0.1 \%$ [1]. Lung torsion occurs when a lobe (or the complete lung) rotates around hilar structures. The degree of rotation is usually $180^{\circ}$, although in some instances, $90^{\circ}$ or $360^{\circ}$ torsion can occur. The torsive lung creates a trifold compromise in airways, arterial blood supply, and in the venous lymphatic drainage [2] due to lumen kinking. The affected lobe often shows signs of hemorrhagic infarction or necrosis. Most torsion cases are detected late and as such are associated with significant mortality [3]. Early recognition with prompt reoperation is the only option for salvaging the remaining lung.

\section{Case}

A 77-year-old woman with a known case of chronic obstructive pulmonary disease presented with symptoms of respiratory tract infection. Her symptoms did not improve despite medical treatment. A chest x-ray was ordered, which resulted in findings in her left lower lobe. This prompted a follow-up with a computed tomography (CT) scan of the chest, which showed a $3.9 \times 4.9 \mathrm{~cm}$ mass in the superior segment of her left lower lobe (Figure 1), and no enlargement of hilar or mediastinal lymph nodes. A CT scan guided biopsy confirmed the presence of malignancy suggesting squamous cell lung cancer. For staging purposes, a positron emission tomography (PET) scan was performed and showed high uptake in the mass and head, while CT scan was normal.
After this, the patient consented to an open left lower lobectomy. Intraoperatively, the tumor appeared clearly on the left upper lobe, but also appeared quite intimate with the ongoing interlobar pulmonary artery of the left lower lobe. We decided that the lingular branch of the pulmonary artery should be divided, followed by division of the ongoing interlobar of the left lower lobe. This would help us obtain gross clearance of the tumor and preserve the remaining arterial supply of the left upper lobe, to be followed by a formal left lower lobectomy. The patient's postoperative course was initially unremarkable, except for a large air leak. She had a postoperative chest radiograph revealing an expanded left upper lobe with chest tubes in place (Figure 2).

On postoperative day 3 , we removed her basal chest tube, after which she developed sinus tachycardia and a sudden cessation of the air leak. This was followed by mild hypoxia, which required $1 \mathrm{~L}$ of oxygen to maintain (oxygen saturation around 90\%). A chest radiograph showed volume loss on the left, as well as whiteout lung and new left sided effusion (Figure 3). Her leukocyte count was raised to 16 and her hemoglobin dropped from $9 \mathrm{~g} / \mathrm{dL}$ to $6 \mathrm{~g} / \mathrm{dL}$. Apical chest tube was functioned with 400 cc serous fluid over 24 hours. Bronchoscopy revealed a complete occlusion of the left upper bronchus with intact left lower lobe stump. The patient was taken to an operating room, where complete torsion of the left upper lobe was confirmed. Torsion was reduced with re-expansion of the lobe. Bronchoscopy confirmed a patent airway and we fixed the lung to the pericardial fat (Figure 4) with linear Ethicon stapler $30 \mathrm{~mm}$. 
The patient recovered in the intensive care unit on positive pressure on a ventilator for 1 day, and on postoperative day 3 , she developed sudden chest pain associated with tachycardia, fever, and high leukocyte count. Chest $\mathrm{x}$-ray showed consolidated left lung with minimal air space. Bronchoscopy and CT scan confirmed presence of retorted left upper lobe (Figure 5). Patient was reexplored, and a 90 degree torsion and non-viable left upper lobe were found (Figure 6). Completion pneumonectomy for left upper lobe was carried out. The patient did well and was discharged after 10 days without any complications.

\section{Discussion}

Lobar torsion represents a rotation of the bronchovascular pedicle with a resultant airway obstruction and vascular compromise. Epplen and Jacobson are credited with the first description of lobar torsion in 1930 [1]. The overall

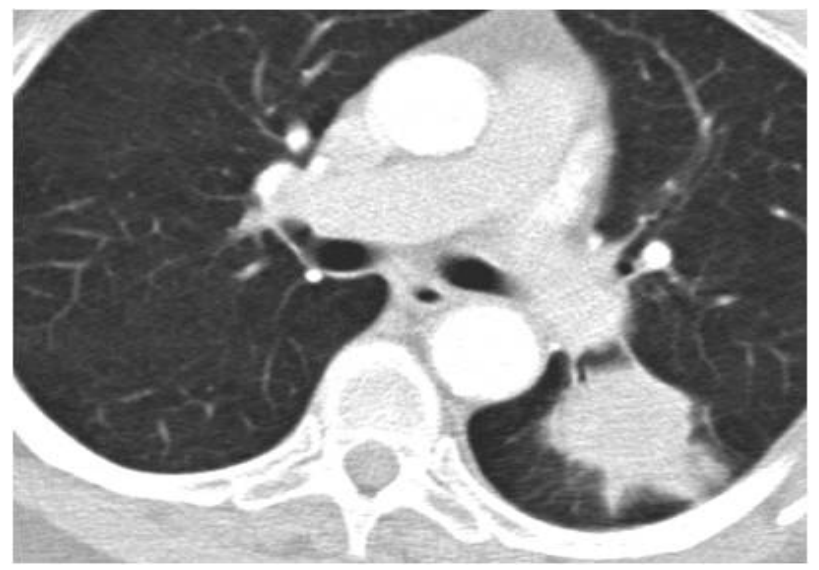

Figure 1. $3.9 \times 4.9 \mathrm{~cm}$ mass in patient's superior segment of the left lower lobe.

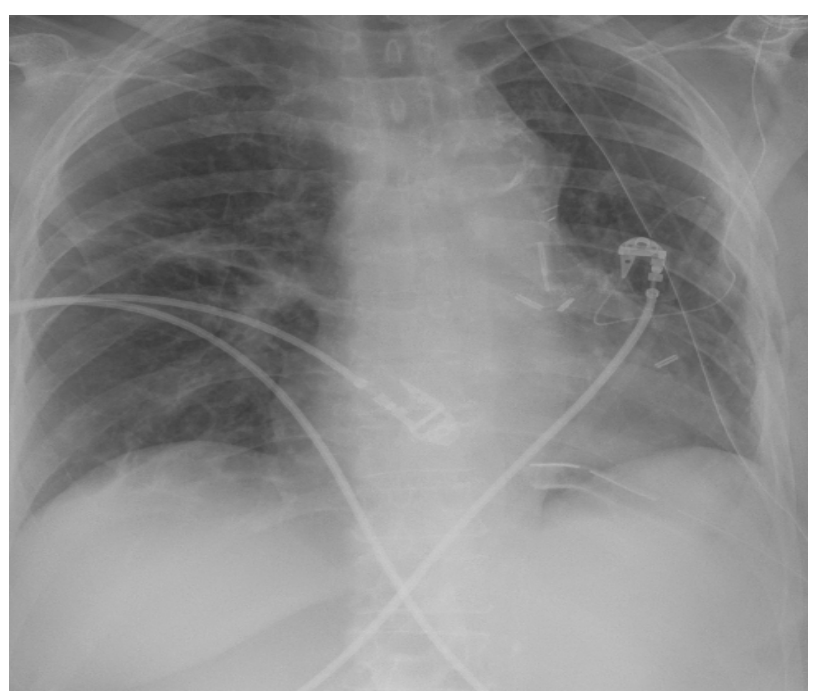

Figure 2. Immediate postoperative chest $x$-ray showing good left upper lobe expansion.

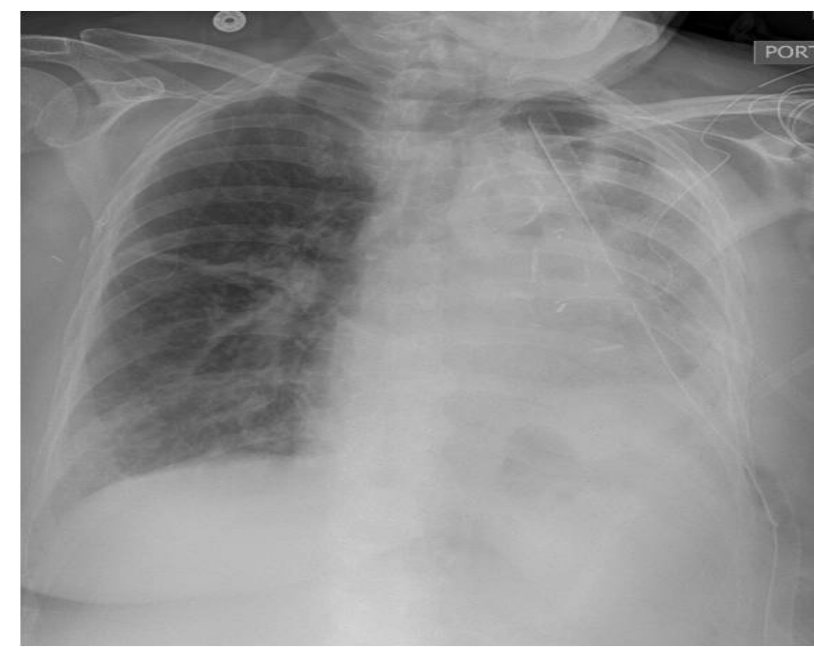

Figure 3. Postoperative day 3 chest radiograph showing volume loss on the left, as well as whiteout lung and new left sided effusion.

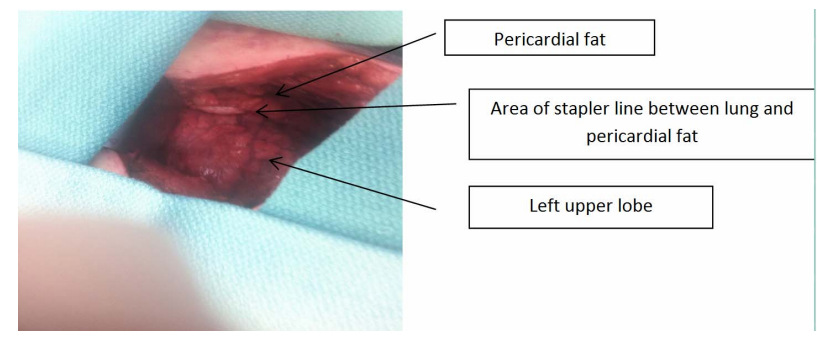

Figure 4. Photo showing fixation of left upper lobe to the pericardial fat.

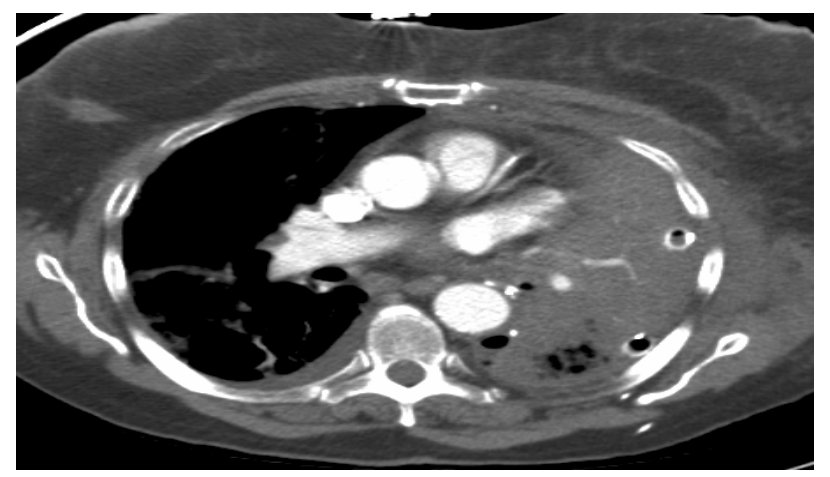

Figure 5. CT scan showing consolidated left upper lobe with element of hypoperfusion.

incidence of lung torsion has been reported as $0.089 \%$ $0.2 \%$ [3]. Left upper lobe torsion is relatively uncommon, compared with right middle or lower lobe torsion. A 70\% chance of lung torsion occurs after right upper lobectomy and only $15 \%$ of the time after left upper lobectomy.

Diagnosis of lung torsion can be difficult due to the rarity of this condition. When diagnosis does occur, it is generally too late to salvage the affected lung parenchyma, contributing to a significant morbidity and mortality rate, especially in patients with borderline pulmonary func- 


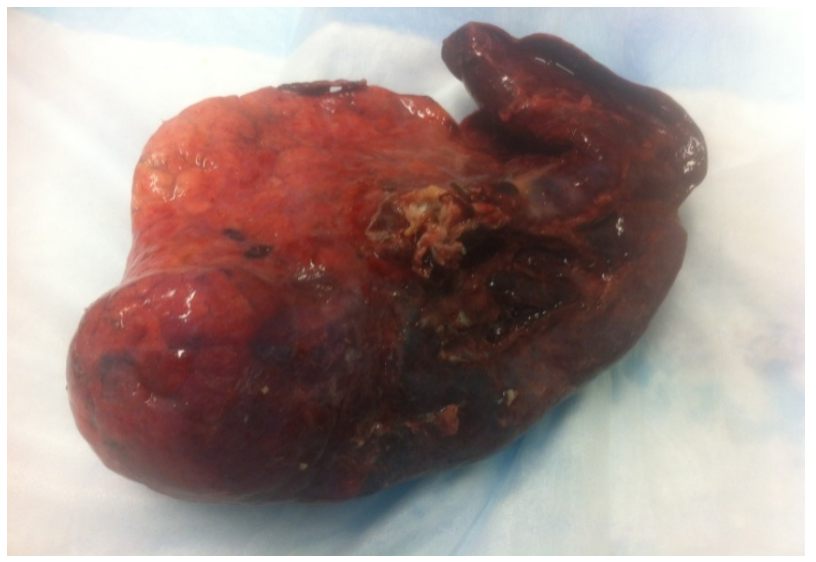

Figure 6. Photo of gangrenace lobe (hailer surface).

tiontests [4]. Cable and colleagues described 7 cases of torsion out of 7887 resections, and all torsion cases in this study required pulmonary resection [3]. Wong and Gold straw reported that $30 \%$ of thoracic surgeons had seen one or more cases of pulmonary torsion, and that most of these instances involved middle lobe torsion after a right upper lobectomy [5].

Approximately 35\% of thoracic surgeons are reported to not routinely fix the middle lobe to the remaining lobe after an upper or lower lobectomy. In this case, we assume removal of a basal chest tube leads to acceleration of left pleural fluid accumulation in presence of minimal pneumothorax, and transaction of interlobar artery and dividing of the lingular branch, to create a perfect environment for a partially free left upper lobe to rotate around the bronchovascular pedicle. Consolidated heavy lobe and fixation of the lung to redundant pericardial fat lead to incomplete torsion in the second event. We did not undertake fixation of the upper lobe in the primary resection for 2 reasons: the upper lobe expanded well during the inflation test, and we planned to decrease the lower lobe dead space after lower lobectomy.

In 1987, Felson noted the mechanisms of torsion underlying circumstances that favor the development of lung torsion [6]; including an airless space, long free lober pedicle, the presence of a parenchymal bridge between contiguous lobes, pneumothorax and pleural effusion, heavy compact lung (due to atlectasis, consolidation or tumors), and transaction of the pulmonary artery. While trauma and thoracic surgery are the most common inciting events, other contributing conditions include spontaneous pneumothorax, pneumothorax induced by percutaneous needle aspiration, diaphragmatic hernia, presence of accessory lobe, pneumonia, neoplasm, and pleural effusion [7].

The findings in this case indicated lung torsion: sudden onset, mild hypoxia not correlated with complete opacification of the affected lung, decrease air entry in the affected lung, sudden cessation of large air leak, tachycardia, drop in hemoglobin, and leukocytosis. With these findings and a high index of suspicion, plus brochoscopic findings of occluded upper lobe bronchus, we decided to operate on this patient without any further tests. In general, presenting signs and symptoms for torsion are non-specific, and include acute chest pain, diminished breath sound over the involved area, copious secretion that may be bloody, hypoxemia, fever, hypotension, and cardiovascular collapse.

Radiologic findings include a change in position of an opacified lobe, alteration or invasion of pulmonary vasculature, a collapsed or consolidated lobe that occupies an unusual position on plain radiograph, and; cutoff; or distortion of bronchus [6,8].

Chest CT scan may show the interruption of bronchus at the level of torsion. Contrast pulmonary angiography during helical CT scanning has the advantage of direct evidence of stenosis or complete obstruction of kinking vessels [9].

Options for surgical intervention include simple detorsion or re-section of the involved pulmonary segments [3]. De-torsion alone is advocated only in patients who undergo re-intervention within a few hours of the primary procedure, while in the majority of patients, pulmonary resection is mandatory due to pulmonary gangrene. Embolisms to other vital organs are the main complication after surgery, which should therefore be carefully monitored [10].

\section{Conclusion}

We suggest that lobar torsion after lobectomy has a low incidence of occurrence, and that early recognition is based on a high index of suspicion followed by aggressive diagnostic evaluation. To minimize torsion, the position of remaining lung lobes should be noted, and should be fixed together or to fixed structures if they are unusually mobile. Aggressive chest physiotherapy and early bronchoscopy should be undertaken early in the postoperative course to decrease the incidence of atlectasis. Rethoractomy should be carried out without any delay to reduce the morbidity and mortality associated with lobar torsion following lung resection.

\section{Acknowledgements}

Our sincere gratitude goes to Dr. Madelaine Plourde, M.D., thoracic surgery consultant, Dalhousie University for her helpful collaboration in this work.

\section{REFERENCES}

[1] F. Epplen and A. L. Jacobson, "Twisted Pedicle of Accessory Lobe of the Lung,” JAMA, Vol. 94, No. 15, 1930, p. 1135.

http://dx.doi.org/10.1001/jama.1930.27120410002009b 
[2] J. C. Nguyen, J. Maloney and J. P. Kanne, "Bilateral Whole-Lung Torsion after Bilateral Lung Transplantation,” Journal of Thoracic Imaging, Vol. 26, No. 1, 2011, pp. W17-W19. http://dx.doi.org/10.1097/RTI.0b013e3181e35ab5

[3] D. G. Cable, C. Deschamps, M. S. Allen, D. L. Miller, F. C. Nichols, V. F. Trastek and P. C. Pairolero, "Lobar Torsion after Pulmonary Resection: Presentation and Outcome," The Journal of Thoracic and Cardiovascular Surgery, Vol. 122, No. 6, 2001, pp. 1091-1093. http://dx.doi.org/10.1067/mtc.2001.117839

[4] S. Hennink, M. W. Wouters, H. M. Klomp and P. Baas, "Necrotizing Pneumonitis Caused by Postoperative Pulmonary Torsion," Interactive CardioVascular and Thoracic Surgery, Vol. 7, No. 1, 2008, pp. 144-145. http://dx.doi.org/10.1510/icvts.2007.158378

[5] P. S. Wong and P. Goldstraw, "Pulmonary Torsion: A Questionnaire Survey and a Survey of the Literature," The Annals of Thoracic Surgery, Vol. 54, No. 2, 1992, pp. 286-288.

http://dx.doi.org/10.1016/0003-4975(92)91386-N
[6] B. Felson, Radiology, Vol. 162, No. 3, 1987, pp. 631-638.

[7] C. Raynaud, S. Lenoir, R. Caliandro, L. Raffenne, P. Validire and D. Gossot, "Spontaneous Middle Lobe Torsion Secondary to Pleural Effusion,” Chest, Vol. 136, No. 1, 2009, pp. 281-283. http://dx.doi.org/10.1378/chest.08-2373

[8] T. Ternes, M. Trump, M. R. de Christenson, G. Howell and J. Stewart, "Spontaneous Middle-Lobe Torsion," Radiology Case Reports, Vol. 8, 2012, p. 812.

[9] D. L. Spizarny, P. C. Shetty and J. W. Lewis Jr., "Lung Torsion: Preoperative Diagnosis with Angiography and Computed Tomography," Journal of Thoracic Imaging, Vol. 13, No. 1, 1998, pp. 42-44. http://dx.doi.org/10.1097/00005382-199801000-00010

[10] E. Apostolakis, E. N. Koletsis, N. Panagopoulos, C. Prokakis and D. Dougenis, "Fatal Stroke after Completion Pneumonectomy for Torsion of Left Upper Lobe Following Left Lower Lobectomy,” Journal of Cardiothoracic Surgery, Vol. 1, 2006, p. 25. http://dx.doi.org/10.1186/1749-8090-1-25 